See discussions, stats, and author profiles for this publication at: https://www.researchgate.net/publication/4163537

\title{
Motor interference between Humans and Humanoid Robots: Effect of Biological and Artificial Motion
}

Conference Paper · August 2005

DOI: 10.1109/DEVLRN.2005.1490951 · Source: IEEE Xplore

\section{CITATIONS}

62

4 authors, including:

Thierry Chaminade

French National Centre for Scientific Research

73 PUBLICATIONS 4,225 CITATIONS

SEE PROFILE
READS

270

David W Franklin

Technische Universität München

89 PUBLICATIONS 4,646 CITATIONS

SEE PROFILE

Some of the authors of this publication are also working on these related projects:

Project Contextual effects in human motor control View project

Project Everyday Activity Science and Engineering (EASE) View project 


\section{Motor interference between Humans and Humanoid Robots:}

\section{Effect of Biological and Artificial Motion}

\author{
Thierry Chaminade \\ ATR Computational Neuroscience Laboratory \\ 2-2-2 Keihanna Science City, Soraku-gun \\ Kyoto, 619-0288, Japan \\ tchamina@atr.jp \\ dfrank@atr.jp
}

\author{
Erhan Oztop \\ Gordon Cheng \\ JST-ICORP Computational Brain Project \\ 2-2-2 Keihanna Science City, Soraku-gun \\ Kyoto, 619-0288, Japan \\ erhan@atr.jp \\ gordon@atr.jp
}

\begin{abstract}
If humanoid robots are to become commonplace in our society, it is important to understand how they are perceived by humans. An influent model in social cognitive neuroscience posits that in human face-to-face interaction, the observation of another individual performing an action facilitates the execution of a similar action, and interferes with the execution of different action. In one interference experiment, null interference was reported when subjects observed an industrial robotic arm moving at a constant velocity perform an incongruent task, suggesting that this effect may be specific to interacting with other humans. This experimental paradigm was adapted to investigate how humanoid robots interfere with humans. Subjects performed rhythmic arm movements while observing either a human agent or humanoid robot performing either congruent or incongruent movements with comparable kinematics. The variance of the executed movements was used as a measure of the amount of interference in the movements. In a previous report, we reported that in contrast to the robotic arm, the humanoid robot caused a significant increase of the variance of the movement during the incongruent condition. In the present report we investigate the effect of the movement kinematics on the interference. The humanoid robot moved either with a biological motion, based on a realistic model of human motion, or with an artificial motion. We investigated the variance of the subjects' movement during the incongruent condition, with the hypothesis that it should be reduced for the artificial movement in comparison to the biological movement. We found a significant effect of the factors defining the experimental conditions, congruency and type of movements' kinematics, on the subjects' variation. Congruency was found to have the expected effect on the area, but the increase in incongruent conditions was only significant when the robot movements followed biological motion. This result implies that motion is a significant factor for the interference effect.
\end{abstract}

Index Terms human-humanoid robot interaction; robot design; social robotics

\section{INTRODUCTION}

Recent advances in humanoid robotics [1-3] bring new social issues into consideration. It is generally accepted that humanoids will become part of our daily lives, as have computers, the internet and email. If we expect their interactions with humans to be natural [4], it is important to study how we perceive humanoid robots. There are some studies addressing the question of what kind of form [5] and functionality [6-8] a human-like robot should have in order to be socially accepted. The former relies on introspective judgments to report how human-like are the robots perceived.
The latter approach relies on the implicit assumption that if a robot has similar cognitive mechanisms as humans (e.g. gaze following) then it must be readily accepted as humanlike, and mainly focus on building such robotic systems.

Conversely, The "uncanny valley" hypothesis proposes that artificial agents imperfectly attempting to impersonate humans induce a negative emotional response [9]. Japanese roboticist Masahiro Mori carried out the earliest investigations on the social competence of artificial anthropomorphic agents [9]. He proposed that as a robot is made more humanlike in its appearance and motion, the emotional response of human subjects towards that robot should increase. Robots that imperfectly attempt to impersonate humans' appearance and motion would, however, trigger a negative response. The emotional response towards a robotic design plotted against its anthropomorphic features would thus have a local minimum close to the maximum similarity to human - a real human being. He dubbed this chasm, which disrupts the linear relation between human likeliness and emotional response, the "uncanny valley of eeriness". This hypothesis predicts, for example, that a prosthetic limb covered with skin-colored rubber, which imperfectly, albeit extremely closely, reproduces the texture and the motion of real limbs would be more repulsive than a less realistic limb with a mechanical appearance. Indeed, the best attempts to create realistic humanoid robots, for example the female robot Actroid, developed by Japanese firm Kokoro, are still unsuccessful in producing sufficient familiarity to avoid the negative emotional response characteristic of this "uncanny valley"; meanwhile, the behavior of the expressive mechanical face Kismet developed at MIT $^{\mathrm{ii}}$ quite effectively engages in intuitive affective and social interactions with humans (see ref. [10] for further examples).

Here we use a new experimental paradigm to test whether a humanoid robot can be perceived as a human. Our paradigm uses an implicit behavioral effect caused by the observation of others' behavior; our results are therefore devoid of the higherlevel cognitive process biases induced when answering introspective questions (e.g. do you think that it is a humanlike movement?). Recent theoretical advances on humans' perception of action and social interactions, reviewed in the next paragraph, propose that the interference between observation and execution of action, used in the present

\footnotetext{
i http://www.kokoro-dreams.co.jp/
}

ii http://www.ai.mit.edu/ 
paradigm, constitutes the automatic aspect of the empathic response. The current paradigm, based on visuo-motor interference, is therefore adapted to the investigation of the automatic and low-level aspects of humans' emotional response to robotic designs.

\section{A. The hypothesis of shared motor representations}

A number of recent studies have demonstrated that common brain areas mediate the perception of others' actions and the execution of actions by the self. Experimental psychology experiments have demonstrated that perceiving a simple action, such as a finger tapping [11], grasping [12], or arm movements [13] facilitates the concomitant execution of the same action and curbs the execution of a different one. These results led to the hypothesis that some cognitive processes are used both in the observation and in the execution of actions. These are termed shared representations.

Similar conclusions were drawn from the study of human brain functions. 'Mirror neurons' were found in reciprocally connected ventral premotor and parietal cortices using monkey electrophysiology. These neurons are activated both when monkeys perform a goal-directed action and when they see the same action performed by an experimenter [14, 15]. In humans, neuroimaging has shown that action-related cortices in the premotor and parietal cortices are activated during observation of actions [16] (see [17] for a computational model).

In addition, ventral premotor and parietal cortices are involved in imitation: In the left hemisphere the premotor cortex appears to be more involved in the goal related aspects of the action [18] whereas the parietal cortex is more involved in body movement [19]. Finally, one study showed that cortices involved in producing a specific action, pointing or writing, are specifically recruited when understanding the goal of another individual's pointing or writing actions [20]. Finally, it has been proposed that motor resonance could explain a number of social behaviors such as contagion of behaviors, synchronicity within a group, and empathy [21].

\section{B. Perception of action and motor interference}

An experimental paradigm was recently introduced to investigate sensory-motor interference in face-to-face behaviors, reproducing a socially valid interaction [13]. It taps into implicit perceptual processing [22], which therefore can be used to test without introspective questionnaires how humanly a robot is perceived by humans. In this paradigm, subjects and the experimenter were face to face, and both produced arm movements that were either horizontal or vertical. Experimental conditions were defined by the direction of the movement and the spatial compatibility between the movements of the subjects and of the other agent's. Movements are compatible when both produce horizontal or vertical movements, and incompatible in the other case.

Variance in the movement was increased when subjects visually follow incompatible movements of human demonstration. However, this effect was specific to watching human actions, and could not be reproduced by the observation of robot actions [13]. In contrast, we have recently shown that a humanoid robot with biologically realistic motion can indeed cause this interference effect [23]. Our current experiment is aimed at revealing whether the form (machine-like vs. human-shape) or the motion (artificial vs. biological) of the robot was essential for the results we have obtained in [23]. If the form is the main factor then we should see the interference effect even when our humanoid robot produces artificial movements. Therefore, current study together with our earlier study [23] when contrasted with [13] can help uncover whether this interference is due to the knowledge of the human nature of the other agent, or due to an automatic processing of certain features of the stimuli (human-like) form and (biological) motion.

\section{HYPOTHESES}

Under the hypothesis that there is a shared substrate between perceiving and executing actions, it is predicted that observing an action should facilitate the execution of the same action, and curb the execution of a different action; a strong interference effect, defined by the increase of variance of the movement, is found when subjects watch an arm movement incompatible to the one they are producing (e.g. vertical versus horizontal) [13]. Interestingly, Kilner et al.'s study did not find any interference effect using a robot. They concluded that the visuomotor interference is specific to interactions between humans [13]. In a previous experiment [23] we tested this claim using a humanoid robot, that is, its general shape is similar to the shape of a human; and the movements of the robot were reproductions of the movements of one of the experimenters. The form and motion of the robot and of the experimenter are similar.

In that case, human-humanoid interactions produced qualitatively comparable but quantitatively different interference effects to human-human interactions. We concluded that the interference effect is explained by an implicit perceptual mechanism sensitive to the general humanoid form and biological motion of the other agent. In addition, the variance doubled in incompatible compared to compatible conditions during human-human interaction, and was multiplied by 1.5 for human-humanoid interaction [23]. In the original report using an industrial robotic arm, the congruency between the subjects' and the industrial robotic arm has no effect on the variance of the subjects' movements. Therefore the interference effect, measured as the ratio between the variance of the movements in the incompatible and compatible conditions, can be used to evaluate the quality of the interaction between humans and artificial agents.

The goal of the present experiment is to test the role of motion in this interference mechanism. We attempt to reproduce the interference effect with the humanoid robot, where the movements are either based on biological or artificial kinematics. Biological kinematics was based on OPTOTRAK recordings of a human's horizontal and vertical movements. These trajectories were used as target for the robot's movements. Artificial kinematics of the movements was produced running a sine wave at a single degree of freedom of the humanoid robot's shoulder, keeping all other degrees of freedom constant. Testing for the existence and the intensity of the interference effect in the artificial motion condition will help us define the involvement of motion characteristics in motor resonance. 


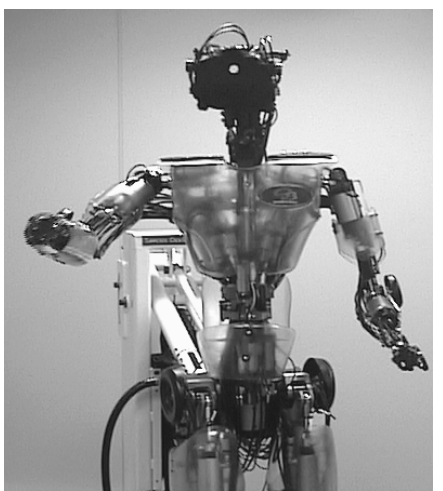

Fig. 1. DB, the humanoid robot used in our experiment

\section{METHODS}

\section{A. The humanoid robot}

We used the humanoid robot $D B$ [3] to produce horizontal and vertical reaching movements. $D B$ is a hydraulic anthropomorphic robot with legs, arms (with hands without fingers), a jointed torso, and a head (Fig. 1). DB was designed by the Sarcos company and the Kawato Dynamic Brain Project, and built by Sarcos to be 1.85 meters tall with a human-like appearance. The robot contains 25 linear hydraulic actuators and five rotary hydraulic actuators having 30 degrees of freedom: three in the neck, two in each eye, seven in each arm, three in each leg, and three in the trunk (Fig. 1).

Our interest was to use the robot's right arm to produce horizontal and vertical movements, thus we commanded only the right arm and the torso joints (for the artificial movement case only the shoulder joint was used) to generate the movement. The robot was mounted from the back eliminating the need to deal with balancing issues. The task of the robot was to replicate the end point Cartesian trajectories recorded from a human demonstrator (Fig. 2; see next section for the data collection details) which were periodic horizontal $(\mathrm{H})$ and vertical (V) reaching movements involving elbow, shoulder and some torso movements. The controller is implemented on the real-time operating system VxWorks using several parallel Motorola PowerPC processors in a VME rack within the environment provided by SL Simulation and Real-Time Control Software Package (http://wwwclmc.usc.edu/publications/S/schaal-TRSL.pdf).

To produce trajectories for the robot, we applied a linear transformation such that the points in the trajectory lie within the workspace of the robot as much as possible. Not all the points were reachable, so the extent of the movements was scaled by 0.9 in both directions allowing the robot to move within its joint limits.

A velocity-based Cartesian controller was used to track the transformed trajectories shown in Fig. 2. The control scheme we used is shown in fig. 3 where the inverse kinematics is solved using Jacobian pseudo-inverse with null space optimization [24]. We considered the tracking achieved satisfactory for our purposes as the robot movements were smooth and human-like for a human observer. Figure 4 shows the tracking in the $\mathrm{x}$ coordinate for the two trajectories used in the experiments. Note that the plane of interest was the one spanned by $\mathrm{x}$ and $\mathrm{z}$ axes.
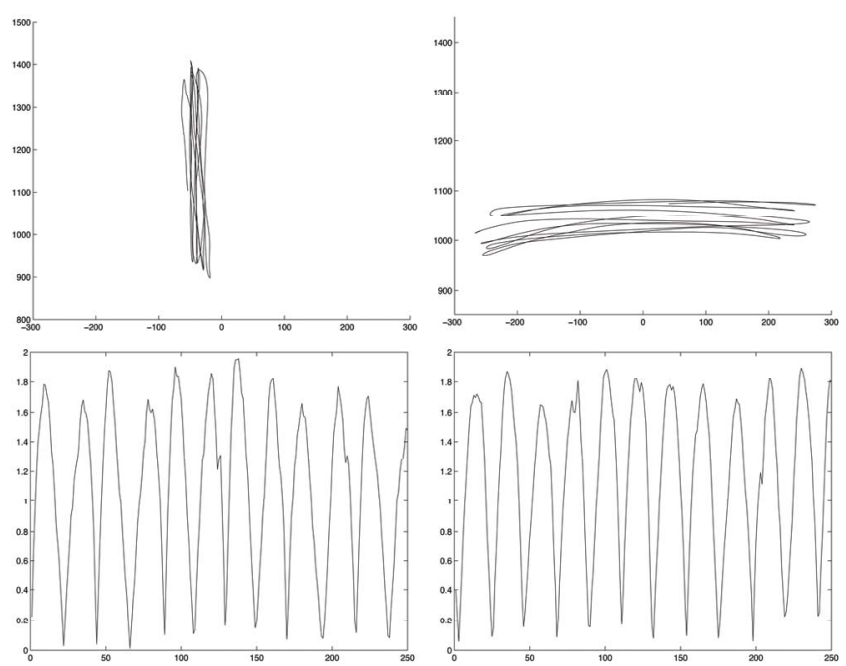

Fig. 2. Example of vertical (left) and horizontal (right) human trajectories, projected onto a vertical plane containing the main axis of the movement, and their respective velocity profiles (bottom), tracked by the robot in the biological motion condition.

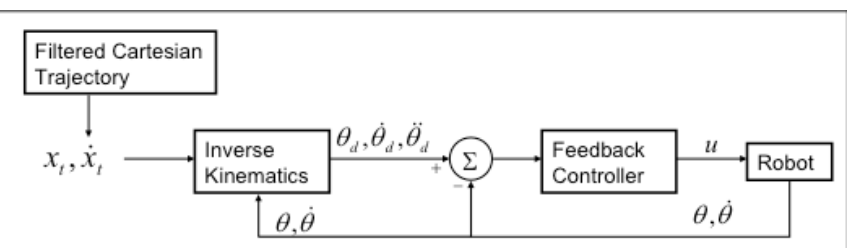

Fig. 3. Cartesian tracker used in generating the arm movements of the humanoid robot.

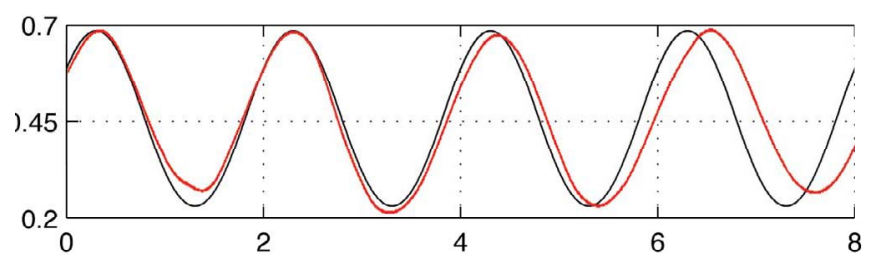

Fig. 4. Example of end-point movement along the vertical $\mathrm{x}$-axis of the robot using biological motion (red line) and the artificial motion (black line) during vertical movements.

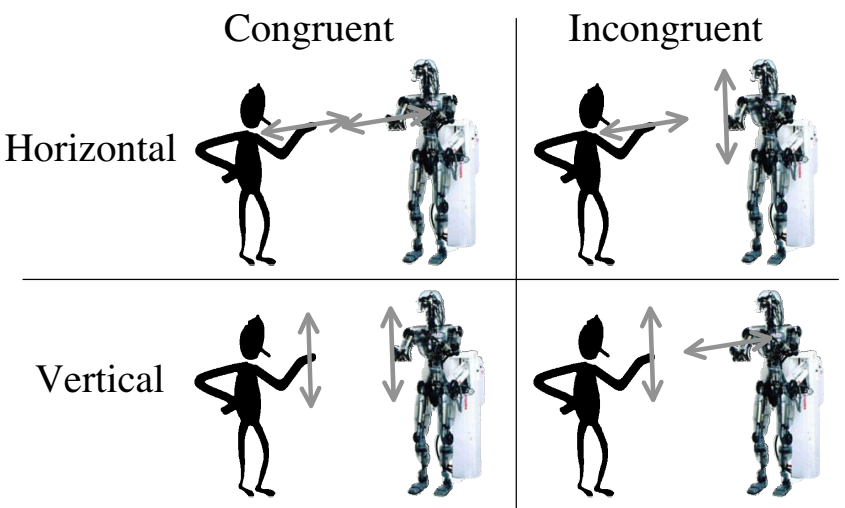

Fig. 5. The Experimental paradigm: subjects executed horizontal (top) vertical (bottom) rhythmic movements. In front of them, a humanoid robot executed either congruent (left) or incongruent (right) movements. The robots movements were either biological or artificial, leading to a $2 \times 2 \times 2$ factorial design. 
Artificial movements were achieved applying a sine rhythmic function to a single degree of freedom of the robot's shoulder joint (flexion-extension for vertical movements, abduction-adduction for horizontal movements). The parameters of each movement (mid-point and span) were set manually to match approximately the average span and location in space of the biological movements. Figure 4 gives an example of biological (red) and artificial (black) movements. Biological movements are characterized by the presence of noise both in the spatial component (e.g. compare the first and second minima of the biological and of the artificial motion) and in the temporal components (e.g. last maxima).

\section{B. Experimental paradigm}

Right-handed naïve subjects performed horizontal $(\mathrm{H})$ or vertical (V) rhythmic arm motions with their right arms in front of their bodies while standing at $0.5 \mathrm{~Hz}$ (Fig. 5).

Subjects performed these movements while standing approximately 2 meters away from a humanoid robot performing similar arm movements (Fig. 5). During each trial, the subjects were instructed to produce one of the two movements $(\mathrm{H})$ or $(\mathrm{V})$ while seeing the robots' arm movements. The movements produced by the subjects and the robots were either spatially congruent ( $\mathrm{C}$; same direction) or incongruent (I; perpendicular). The subjects were also instructed to be in phase with the robot's movements. For each 30 second trial, the kinematics of the endpoint of the subject's right index finger was recorded at $210 \mathrm{~Hz}$ using the OPTOTRAK 3020 system (Northern Digital, Waterloo). Examples of trajectories, projected on the vertical main plane of the movement, and velocity profiles, are given in Fig. 2 .

The experiment contained 8 conditions $((\mathrm{R} / \mathrm{L}) \mathrm{x}(\mathrm{C} / \mathrm{I}) \mathrm{x}$ (B/A)). In addition, 4 templates were used for biological motion, leading to a total of 32 different experimental conditions. These conditions were performed in a random order of presentation, in the form of two sessions of 16 conditions.

\section{Analysis}

The three dimensional trajectories of each marker were digitally low-pass filtered using a zero-lag, fifth-order Butterworth filter with a cut-off frequency of $25 \mathrm{~Hz}$. Each movement was segmented from the surrounding movements using the 3D curvature[25] (c(t)) where:

$$
\mathbf{c}(\mathbf{t})=\frac{\sqrt{\|\dot{\mathbf{x}}(\mathbf{t})\|^{2}\|\ddot{\mathbf{x}}(\mathbf{t})\|^{2}-\left(\dot{\mathbf{x}}(\mathbf{t})^{\mathbf{T}} \ddot{\mathbf{x}}(\mathbf{t})\right)^{2}}}{\|\dot{\mathbf{x}}(\mathbf{t})\|^{3}}
$$

This measure of curvature is very low when the velocity of the movement is high, but becomes very high as velocity slows and the movement changes direction. The beginning and end of each movement was removed if the curvature was above $100[1 / \mathrm{m}]$ preventing small drifts in the hand location at the extremes of movement from influencing the results.

By using the measure of curvature, each condition was segmented into 10 upward and 10 downward movements for further analysis. The three dimensional kinematic data of the finger was projected onto a vertical plane containing the main axis of the movement (Vertical) and an orthogonal plane (Horizontal).
In order to quantify the effects of the conditions on the behavior of the subjects, we calculated the signed area of each movement defining the deviation from the straight-line joining the start and end of each segmented movement [26]. This area was calculated separately in the vertical and horizontal planes. A 6-ways ANOVA (Subject $x$ Type of motion $[\mathrm{B} / \mathrm{A}] \mathrm{x}$ Congruency $(\mathrm{C} / \mathrm{I}) \mathrm{x}$ Movement $(\mathrm{H} / \mathrm{V}) \mathrm{x}$ repetition) was performed on these two values. The ANOVA considered first and second level interaction effects, significant at the alpha 0.05 level. Multiple comparisons were performed using Tukey's honestly significantly different test at the alpha 0.05 level.

\section{RESULTS}

Subjects significantly modified the variables at the threshold used, and are not be reported in the ANOVA tables for the Vertical area (Table 1) and Horizontal area (Table 2).

\begin{tabular}{lccc}
\multicolumn{4}{c}{ TABLE 1: ANOVA ON THE VERTICAL AREA. } \\
Source & $\mathbf{d f}$ & $\mathbf{F}$ & $\boldsymbol{p}$ \\
Type & 1 & 1.65 & 0.1987 \\
Congruency & 1 & 21.03 & $<0.05$ \\
Movement & 1 & 7983.29 & $<0.05$ \\
Repetition & 3 & 10.18 & $<0.05$ \\
Type x Congruency & 1 & 9.90 & $<0.05$ \\
Type x Movement & 1 & 40.61 & $<0.05$ \\
Type x Repetition & 3 & 9.02 & $<0.05$ \\
Congruency x Movement & 1 & 0.10 & 0.7564 \\
Congruency x Repetition & 3 & 6.64 & $<0.05$ \\
Movement x Repetition & 3 & 1.19 & 0.3241 \\
& & & \\
Source & TABLE 2: ANOVA on THE HORIZONTAL AREA. & \\
Type & $\mathbf{d f}$ & $\mathbf{F}$ & $\boldsymbol{p}$ \\
Congruency & 1 & 83.54 & $<0.05$ \\
Movement & 1 & 40.51 & $<0.05$ \\
Repetition & 1 & 134.10 & $<0.05$ \\
Type x Congruency & 3 & 12.07 & $<0.05$ \\
Type x Movement & 1 & 12.09 & $<0.05$ \\
Type x Repetition & 1 & 15.97 & $<0.05$ \\
Congruency x Movement & 3 & 13.80 & $<0.05$ \\
Congruency x Repetition & 3 & 94.99 & $<0.05$ \\
Movement x Repetition & 3 & 20.00 & $<0.05$ \\
& & & $<0.05$ \\
& & &
\end{tabular}

There was a significant effect of the experimental conditions defined by the interaction between the type of movement and the congruency. Multiple comparisons between the areas in the four experimental conditions defined by the type of movement and the congruency were performed to further explore the variation of the horizontal and vertical areas, and results are presented on Fig. 6.

\section{DISCUSSION}

\section{A. An objective tool to assess human perception of robots}

Robots designers currently rely on the assumption that anthropomorphic robots would be more competent in social interactions because humans would interact intuitively with them [4]. However this assumption is widely unexplored, 
especially considering the conflicting hypothesis called the 'uncanny valley' introduced by Masahiro Mori [9]. For this roboticist, the relation between human empathic reaction and anthropomorphism of the robot does not show a monotonic increasing curve, but presents a sharp chasm, indicating a strong negative reaction, before reaching the exact humanlikeness. Robots imperfectly attempting to reproduce humans would cause a negative reaction leading to the rejection of the robot as an interacting partner.

Despite the consequences this 'uncanny valley' hypothesis could have in the design of interacting robots, it has not been investigated scientifically, perhaps due to a lack of an objective tool to test the human reaction. Instead, designers usually rely on intuition or surveys, which can suffer from subjective biases. It is thus desirable to create a paradigm in order to assess the human reaction to the perception of robots without conscious introspection.
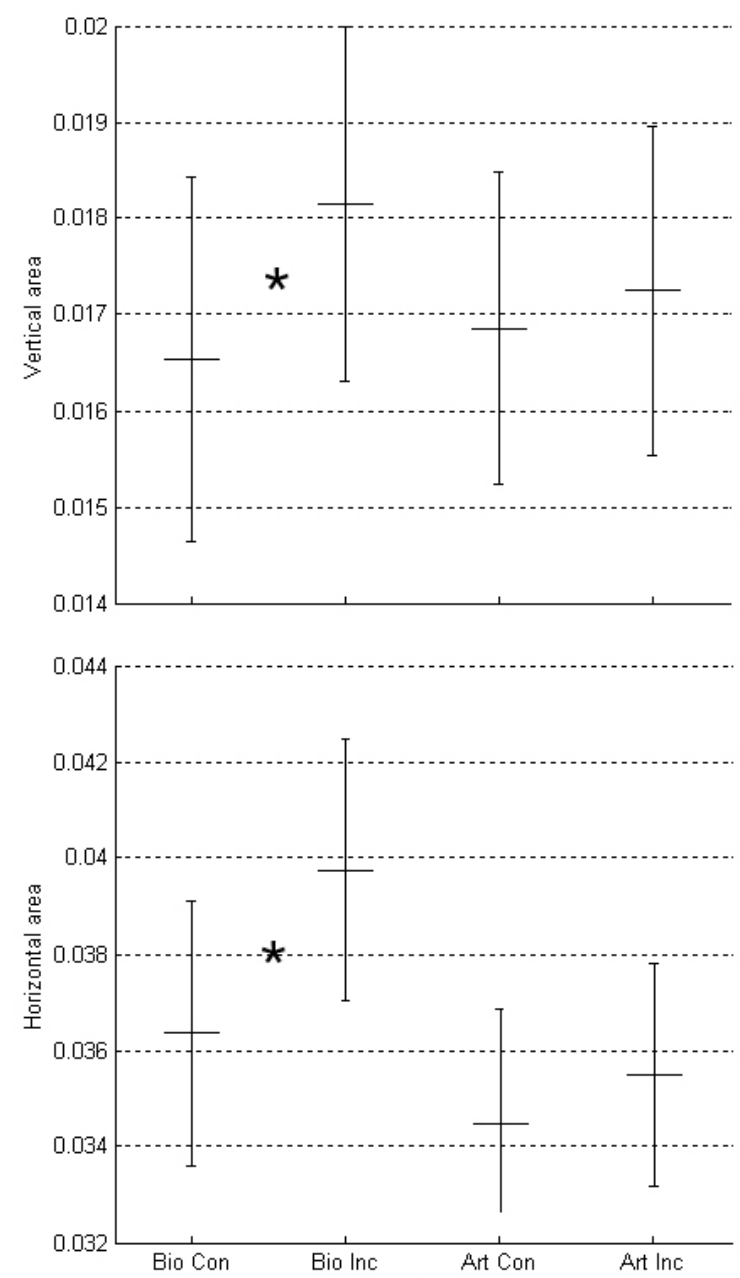

Fig. 6. Mean projected area (error bar: standard error of the means) in each of the four experimental conditions defined by the type of movement (biological: Bio and artificial: Art) used to control the robot and the congruency (congruent: Con and incongruent: Inc). The area was significantly increased between congruent and incongruent movement when the robot was controlled with biological motion (*: Tukey's HSD $p<0.05$ ).
We contend that the experimental framework and tools developed in behavioral sciences to investigate social interactions among humans could be adapted to test human interactions with robots. One prominent hypothesis in cognitive neuroscience is the existence of a common framework for execution and perception of action. Within this framework, we adapted a motor interference study [13], which is well suited for examining full body interactions.

In a previous experiment [23], we found a significant effect of the congruency, described as the variance of the area in the vertical plane, for both interaction with the human agent and the humanoid robot. There is an increase of the variability in the incongruent conditions for both human agent and humanoid robot. The ratio between the incongruent and congruent conditions was higher when subjects interacted with the human agent $(2.1: 1)$ than with the humanoid robot $(1.4: 1)$. In the present experiment, the same procedure was adapted to investigate the effect of motion.

\section{$B$. Effect of motion on interference}

Interference was measured as the variability of the subjects' arm movements while observing congruent and incongruent movements of a humanoid robot which were either biological or artificial. Biological movements were based on human motion capture data, and used as targets for the humanoid robot movements. This biological movement used many degrees of freedom throughout the right arm and the torso of the robot, giving a natural look to the movements. On the other hand, artificial motion were created using sinusoidal shoulder joint movements (1 DOF) giving an unnatural look to the arm's movements. Despite these differences, the end effector's movements in space were very similar in terms of frequency and range of movement.

Subjects' movements were projected onto horizontal and vertical planes under the hypothesis that interference would only be found on the vertical plane, on which congruency was controlled. Fig. 6 shows that the deviation area is significantly increased between congruent and incongruent conditions only for the biological motion, but the small increase for the artificial motion is not statistically different. This shows that form alone is not enough to trigger an emotional response; a realistic motion has to accompany the form for eliciting a response similar to the human movement observation.

\section{CONCLUSION}

The motor interference, which is explained by the shared representation hypothesis, is not specific to human-human interactions but can also be observed in human-humanoid interactions. Collectively these studies [13, 23] suggest that combination of the form and the motion of an agent is an important factor for the social competence of the interaction. The present experiment shows that motion is crucial in this interference, and further studies to tease apart form and motion are underway. It also validates an effective experimental paradigm to assess a human's implicit reaction to a humanoid robot. It should now be extended to investigate and guide the design of socially competent humanoid robots. 


\section{ACKNOWLEDGEMENTS}

We thank Gary Liaw for his assistance in data analysis.

\section{REFERENCES}

[1] B. Adams, C. Breazeal, R. A. Brooks, and B. Scassellati, "Humanoid Robots: A New Kind of Tool," IEEE Intelligent Systems, vol. 15, pp. 25-31, 2000.

[2] G. Cheng, A. Nagakubo, and Y. Kuniyoshi, "Continuous Humanoid Interaction: An Integrated Perspective - Gaining Adaptivity, Redundancy, Flexibility - In One," Robotics and Autonomous Systems, vol. 37, pp. 161-183, 2001.

[3] C. G. Atkeson, J. G. Hale, F. E. Pollick, M. Riley, S. Kotosaka, S. Schaal, T. Shibata, G. Tevatia, A. Ude, S. Vijayakumar, and M. Kawato, "Using Humanoid Robots to Study Human Behavior," IEEE Intelligent Systems, vol. 15, pp. 46-56, 2000.

[4] T. Fong, I. Nourbakhsh, and K. Dautenhahn, "A survey of socially interactive robots," Robotics and Autonomous Systems, vol. 42, 2002.

[5] C. DiSalvo, F. Gemperle, J. Forlizzi, and S. Kiesler, "All robots are not created equal: the design and perception of humanoid robot heads," Proceedings of the conference on Designing interactive systems: processes, practices, methods, and techniques, vol. http://doi.acm.org/10.1145/778712.778756, 2002.

[6] B. Scassellati, "Foundations for a theory of mind for a humanoid robot," B. Scassellati, Foundations for a theory of mind for a humanoid robot, Ph.D. Thesis, Dept. Elec. Eng. Comp. Sci., MIT, 2001., 2001.

[7] H. Kozima and H. Yano, "A robot that learns to communicate with human caregivers," H. Kozima and H. Yano, A robot that learns to communicate with human caregivers, in: Proc. Intl. Wksp. Epigenetic Rob., 2001., 2001.

[8] C. Breazeal and B. Scassellati, "Infant-like Social Interactions between a Robot and a Human Caretaker," CaretakerAdaptive Behavior, vol. 8, pp. 49-74, 2000.

[9] M. Mori, "The valley of eeriness (japanese)," Energy, vol. 7, pp. 33-35, 1970.

[10] K. Dautenhahn, "Design Spaces and Niche Spaces of Believable Social Robots," presented at IEEE ROMAN 2002, Berlin, Germany, 2002.

[11] M. Brass, H. Bekkering, A. Wohlschlager, and W. Prinz, "Compatibility between observed and executed finger movements: comparing symbolic, spatial, and imitative cues," Brain Cogn, vol. 44, pp. 124-43., 2000.

[12] M. G. Edwards, G. W. Humphreys, and U. Castiello, "Motor facilitation following action observation: A behavioural study in prehensile action," Brain and Cognition, vol. 53, pp. 495-502, 2003.
[13] J. M. Kilner, Y. Paulignan, and S. J. Blakemore, "An interference effect of observed biological movement on action," Current Biology, vol. 13, pp. 522-525, 2003.

[14] V. Gallese, L. Fadiga, L. Fogassi, and G. Rizzolatti, "Action recognition in the premotor cortex," Brain, vol. 119, pp. 593-609, 1996.

[15] G. Rizzolatti, L. Fadiga, V. Gallese, and L. Fogassi, "Premotor cortex and the recognition of motor actions," Cognitive Brain Research, vol. 3, pp. 131$141,1996$.

[16] J. Grezes and J. Decety, "Functional anatomy of execution, mental simulation, observation, and verb generation of actions: a meta-analysis," Hum Brain Mapp, vol. 12, pp. 1-19., 2001.

[17] Oztop E, Wolpert D, Kawato M (2005) "Mental state inference using visual control parameters," Cogn Brain Res vol. 22, pp. 129-151, 2005.

[18] L. Fadiga and L. Craighero, "New insights on sensorimotor integration: From hand action to speech perception," Brain and Cognition, vol. 53, pp. 514524, 2003.

[19] M. A. Arbib, A. Billard, M. Iacoboni, and E. Oztop, "Synthetic brain imaging: grasping, mirror neurons and imitation," Neural Networks, vol. 13, pp. 975997, 2000.

[20] T. Chaminade, D. Meary, J. P. Orliaguet, and J. Decety, "Is perceptual anticipation a motor simulation? A PET study," Neuroreport, vol. 12, pp. 3669-3674., 2001.

[21] V. Gallese, "The manifold nature of interpersonal relations: the quest for a common mechanism," Philosophical Transactions of the Royal Society of London: B Biological Sciences, vol. 358, pp. 517528, 2003.

[22] S.-J. Blakemore, J. Winston, and U. Frith, "Social cognitive neuroscience: where are we heading?," Trends in Cognitive Sciences, vol. 8, pp. 216-222, 2004.

[23] E. Oztop, D. W. Franklin, and T. Chaminade, "Human-humanoid interaction: is a humanoid robot perceived as a human," presented at Humanoids 2004, Los Ageles, CA, USA, 2004.

[24] L. Sciavicco and B. Siciliano, Modelling and control of robot manipulators. London ; New York: Springer, 2000.

[25] S. Schaal and D. Sternad, "Origins and violations of the $2 / 3$ power law in rhythmic three-dimensional arm movements," Exp Brain Res, vol. 136, pp. 60-72, 2001.

[26] D. W. Franklin, R. Osu, E. Burdet, M. Kawato, and T. E. Milner, "Adaptation to stable and unstable dynamics achieved by combined impedance control and inverse dynamics model," J Neurophysiol, vol. 90, pp. 3270-82, 2003. 